\title{
Exploration of Digital Frequency Band System
}

\author{
Xiaoping ${ }^{1 a}$ Xu, Anqi Wang ${ }^{2}$ and Weiqi Wang ${ }^{3}$ \\ ${ }^{123}$ Electronic information and control engineering, Beijing university of technology, China
}

\begin{abstract}
Digital modulation techniques is a very important method of realizing modern communication. The showing up of Digital modulation techniques promoted the development of modern communication.. Research on digital communications modulation theory, provide an effective modulation, compressed signal transmission band, increase channel multiplexing efficiency, improve signal transmission reliability, has important significance in practice.
\end{abstract}

\section{Introduction}

Which will mainly explore research is the basic principle of digital band transmission system, using VHDL hardware description language based, MATLAB as an auxiliary tool, developed each module modem programming, and digital band transmission teach development system developed exploration of modulation and demodulation of the digital band systems research.

First, the digital band system into amplitude shift keying (ASK), frequency shift keying (FSK), phase shift keying (PSK), differential phase shift keying (DPSK), and were done for ASK, FSP, PSK, DPSK research. Each system debugging and simulation, thereby drawn from the analysis of digital band modulation process, results and characteristics.

\section{Amplitude shift keying (ASK)}

Digital amplitude modulation is to use digital baseband signal to control the amplitude of the sinusoidal carrier, the signal amplitude of the carrier varies with changes in the baseband signal changes, the most basic digital modulation amplitude modulation techniques include binary amplitude modulation and monitoring ary amplitude shift keying modulation. Fundamentals derivation omitted

Waveform simulation and waveform analysis:

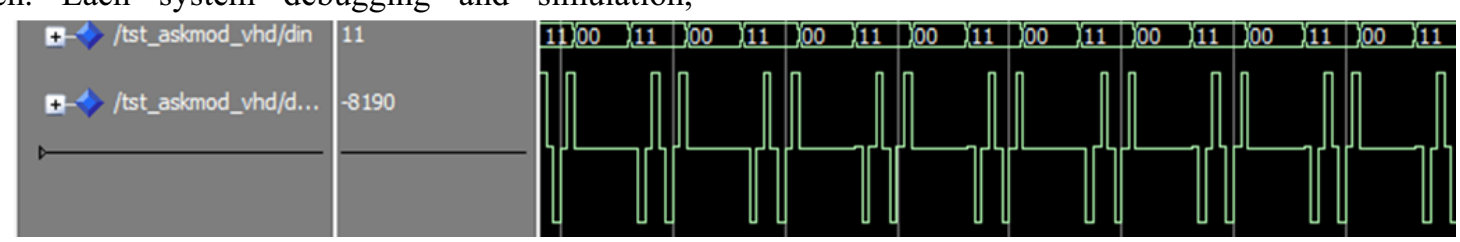

Figure 1 Simulation waveform modulation signal 2ASK 


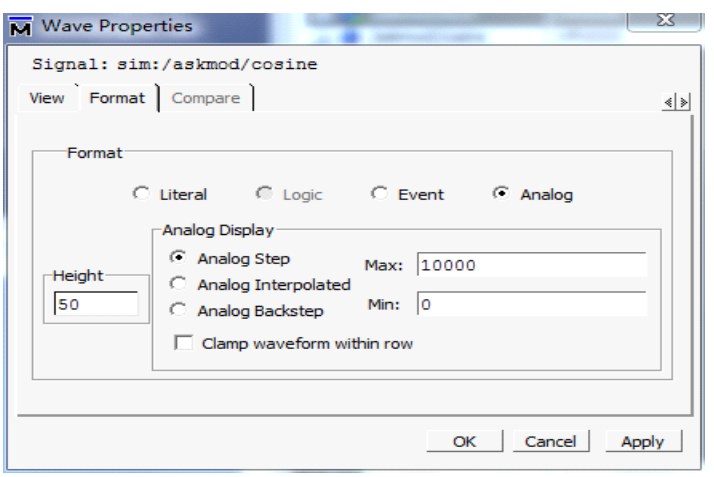

Figure 2 Waveform characteristics setting Figure

2ASK is based on the value of the input pin to adjust the amplitude of the output signal of the cosine signal can be seen from the figure, the output signal having a value of zero and a maximum value in both cases.

4ASK Simulation of the modulation signal waveform :

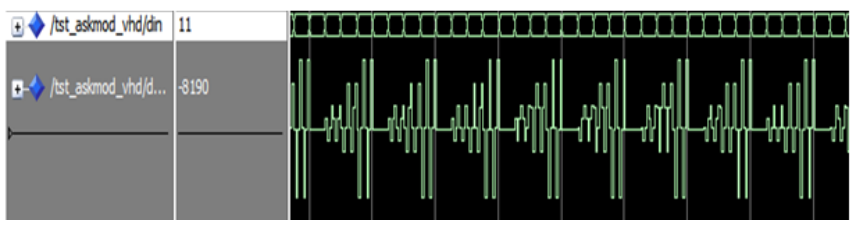

Figure 3 Simulation of the modulation signal waveform

When 4ASK modulation, the input signal is: $00,01,10,11$, the amplitude of the output signal is shown, there are four, maximum, minimum, and the middle two values $0.3281 \mathrm{max}$ and $0.6563 \mathrm{max}$ (approximately $1 /$ $3 \max$ and $2 / 3 \max )$.

Demodulation section: 2ASK demodulated signal (without shaping filter) waveform shown in Figure 4, the input signal $2 \mathrm{ASK}$ rectification and low-pass filtering

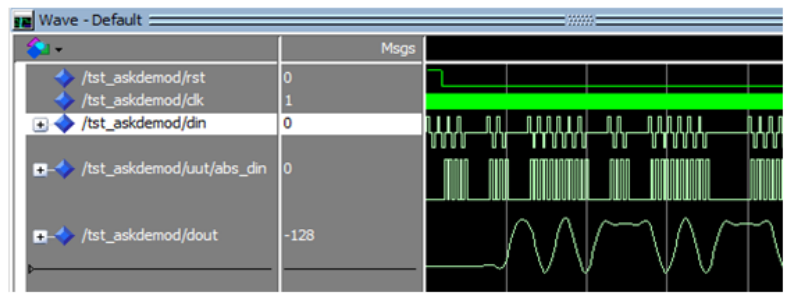

Figure 4 2ASK demodulated signal waveform simulation (without shaping filter)

2ASK demodulated signal (shaping filter) simulation waveforms in Figure 5, after the forming of 2ASK signal filtering, rectifying and low-pass filtering.

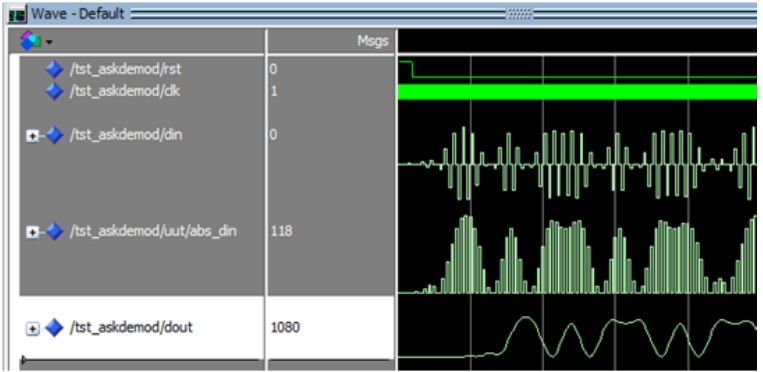

Figure 5 2ASK demodulated signal (shaping filter) simulation waveforms

4ASK demodulation signal (without shaping filter) simulation waveforms in Figure 6, the input signal to 4ASK rectification and low-pass filtering

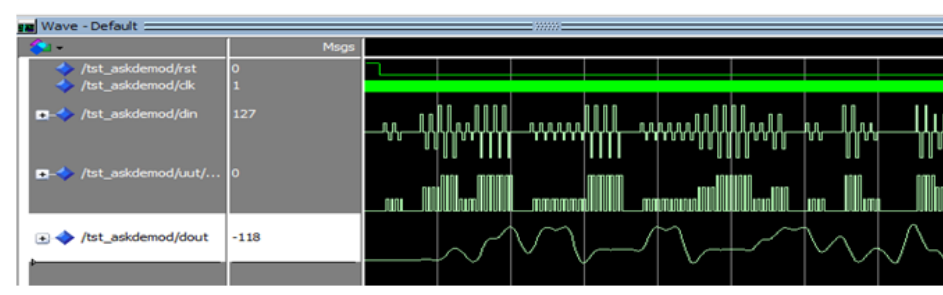

Figure 6 4ASK demodulation signal (without shaping filter) simulation waveforms

4ASK demodulation signal (shaping filter) simulation waveforms in Figure 7, after the forming of 4ASK signal filtering, rectifying and low-pass filtering.

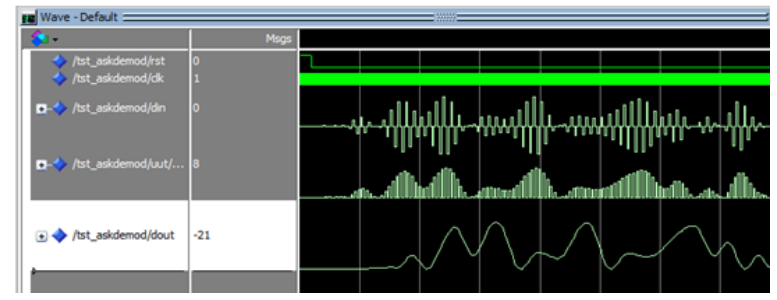

Figure 7 4ASK demodulation signal (shaping filter) simulation waveforms

The results of the simulation results with matlab (Figure 8) were compared and found consistent simulation waveforms, the program can prove ASK signal is demodulated.

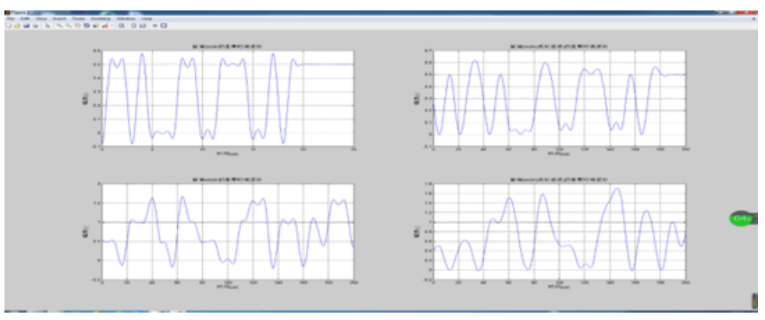

Figure 8 Mat lab for 2ASK, 4ASK demodulation simulation waveform shaping filter.

Frequency shift keying (FSK)

Fundamentals derivation omitted 


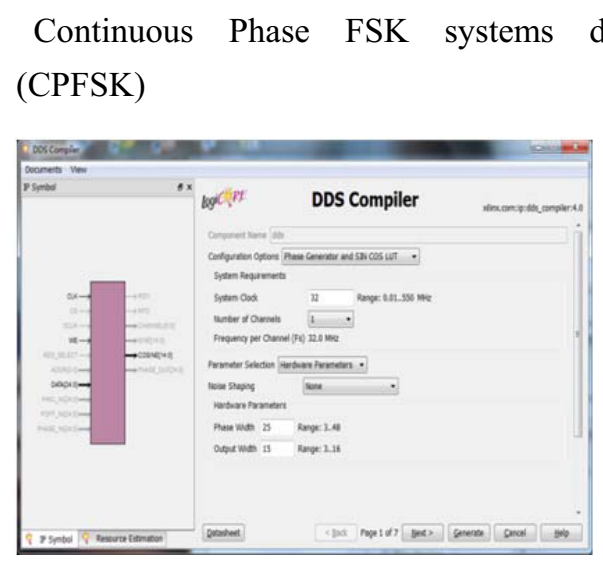

Figure Demodulation aspects of using non-coherent demodulation, design model design as shown in Figure 10.

Figure 9 A part of DDS parameter settings

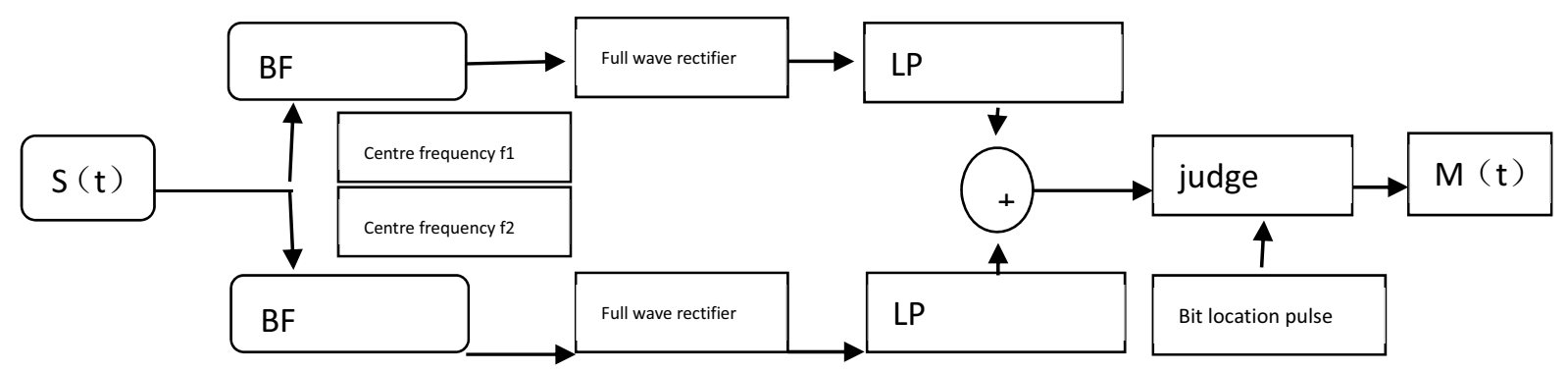

Figure 10 Non-coherent demodulation of the design model

Waveform simulation and waveform analysis

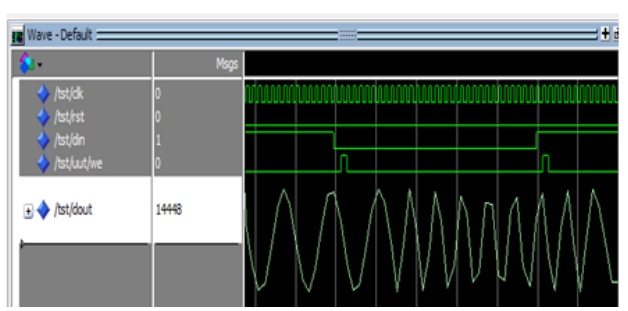

Figure 11 Continuous phase FSK modulation waveform simulation

Use DDS to produce a continuous phase frequency signal, high-frequency serial data clock detection Edge to DDS frequency control port write word after the transition data, the data enable signal is 1 , initialization frequency.

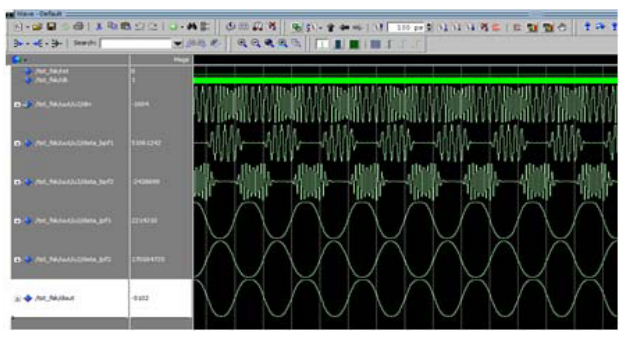

Figure 12 Continuous phase FSK modulation waveform simulation

Modulated output as the demodulation input, through non-coherent demodulation get 12 simulation results. According to the simulation results, see the output signal maintains the continuity of the signal phase.

\section{A phase shift keying (PSK)}

Fundamentals derivation omitted

Four-phase relative phase shift keying (QPSK) system design

It's selected representative DQPSK, four phase relative phase shift keying modulation scheme uses a phase modulation method, primarily shaping filter, serial-parallel conversion, together with a differential coding, bipolar transform. 


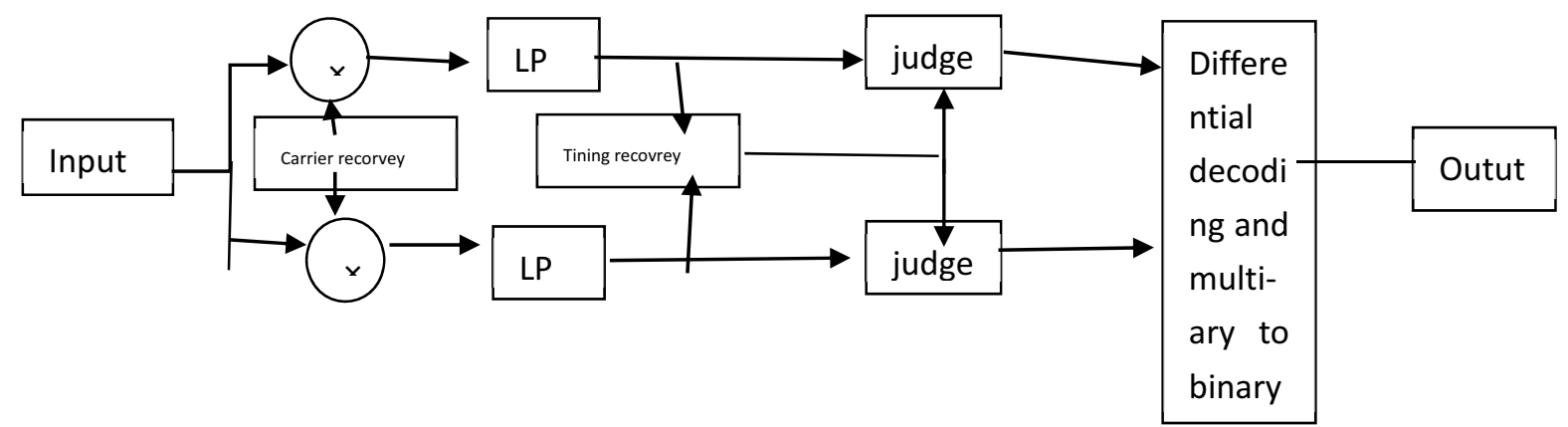

Figure 13 Block Diagram DQPSK coherent demodulation

Waveform simulation and waveform analysis

QPSK modulation waveform shown in Figure 14

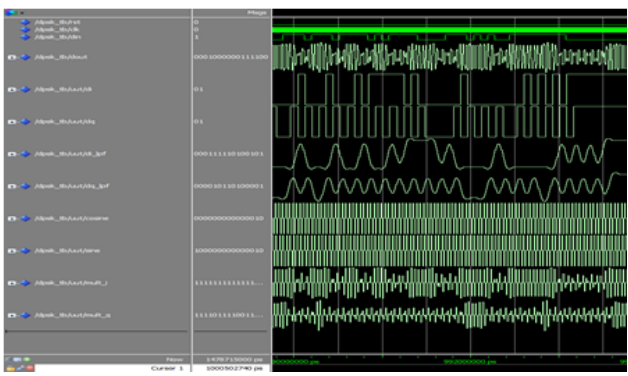

Figure 14 QPSK modulation waveform

QPSK demodulator:First carried out by costas carrier synchronization, demodulating the baseband signal output

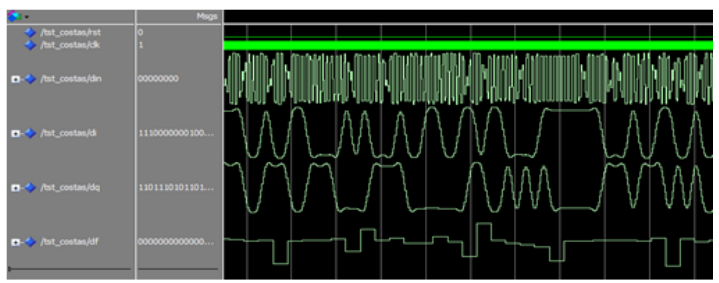

Figure 15 DQPSK demodulator polarity costas loop simulation waveforms

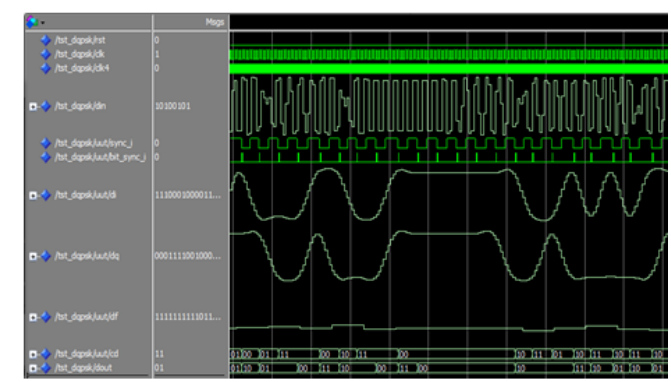

Figure 16 Polar costas loop when no deviation waveform diagram Convergence

Figure 15 were treated through various waveforms generated, three modules were converted code pattern (serial to parallel converter, differential coding, bipolar change), shaping filter, nco module (generated in the same direction orthogonal to the carrier signal and multiplier), a multiplier module, the final in-phase, quadrature branch combined, the output DQPSK signal.
By Costas loop to achieve a coherent carrier extraction, and generates orthogonal to the branch with the baseband waveform.Figure 15 by polar costas demodulated baseband waveform ring

As can be seen from FIG. 16, the data after run to 20,000, after the frequency difference loop convergence jitter significantly reduced, a difference also corresponds to the decrease, increase demodulation efficiency.

Then through BitSync for bit synchronization, were added to the bit timing judgment and differential decoding, then the individual modules are connected through the component is instantiated, it can make the whole demodulation 25

Through simulation overall map view, then loop stability data before and after the DQPSK demodulator exactly.

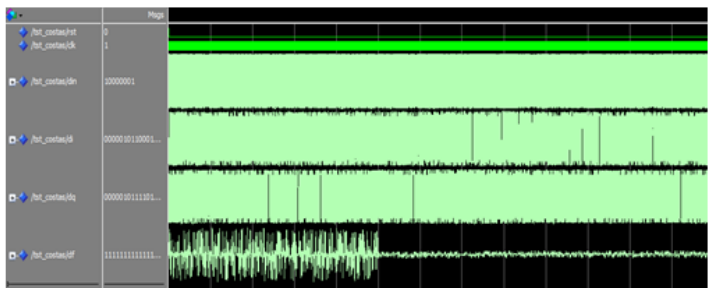

Figure 17 QPSK demodulation system simulation wavefor

\section{Summary}

Through this research and exploration, according to the bandwidth of each modulation system to analyze their anti-noise performance Specifically, look at the binary digital modulation contrast, the rate for data dissemination from PSK $>$ FSK $>$ ASK. ASK worst noise immunity, anti-fading capability is not strong, which generally adapt to constant use in the reference channel, FSK strong noise immunity, easy to implement, PSK in the cable channel to achieve high-speed transmission, but the requirements of a stable receiver the reference phase is used to distinguish between the various phases, 
the best noise immunity, also strong resistance to decay, to implement more complex. The multi-band compared with the binary system improves bandwidth efficiency, but the complexity of the realization of the increase, at the same information rate, multi-band signal element duration wider than the binary, widening the symbol width will increase signal element can also reduce the impact of energy due to inter-symbol interference caused by the channel characteristics of.

\section{References}

1. Junnian Wang,Jinfeng Wu. Based on Matlab /

Simulink communication principle visual dynamic simulation [J]. Gansu Agricultural University 2003(6).

2. Zhixing Zhang, matlab program design and application [M], Tsinghua University Press,2004 